\title{
A combined approach for enhancing smile algorithm- A
} case report

\author{
Ashwith B Hegde*, Wasu Patil, Ajit Kalia, Gupta Gaurav and Sonali Khandekar \\ Department of Orthodontics, M.A.Rangoonwala Dental College, Azam Campus, Camp Pune 411001, India
}

\begin{abstract}
A combined team approach is sometimes required for diagnosis and treatment planning in a patient to get an ideal result. This case report describes the combined approach followed to treat an adult patient with proclina-tion and spacing in the upper and lower anterior teeth with upper peg lateral incisors.

Orthodontic treatment was initiated to correct the inclination and overbite which was followed by prosthodontic restoration of the peg laterals with full ceramic crowns to achieve ideal aesthetics and an improved occlusion.
\end{abstract}

\section{Introduction}

A Hollywood smile is the paramount objective of all patients seeking orthodontic therapy. A problematic summation of missing teeth, proclination, and tooth shape deformity cannot give an ideal treatment outcome using orthodontic treatment alone.

Enhanced smile aesthetics can be achieved in such conditions with an interdisciplinary approach i.e. combining orthodontics with prosthodontics. This treatment approach not only ensures a good aesthetic outcome but also enables good functional occlusion and long term stability of achieved results [1-3].

Bolton tooth size discrepancy sometimes is a major hindering factor in achieving the desired orthodontic results. Tooth size discrepancy is often overlooked by clinicians in their regular orthodontic diagnosis the realisation of which is only understood at the final stages where aesthetics is compromised.

A team approach is sometimes necessary at the initial stage of diagnosis and treatment planning to avoid this dilemma in the final treatment outcome. It is important for an orthodontist to keep himself abreast about the developments in the field of dentistry and applying them to achieve favourable orthodontic outcomes. A Team approach not only enhances the level of beauty but also is a major contributing factor in enhancing the psychological self esteem of the patient.

The current article describes a team approach for achieving excellent results which is the prime requirement of all patients seeking orthodontic therapy.

\section{Case history}

A 15 year old female patient reported to our Orthodontic Office with chief complaint of protruded and spaced upper front teeth. She did not give any other relevant history. She was conscious of her small tooth, protrusion and smile (Figure 1).

Intraoral Clinical Examination revealed Angles class I molar with proclined and spaced upper incisors. A closer evaluation of the dentition revealed peg shaped upper lateral incisors on both the sides.
Extraoral Clinical Examination exhibited convex profile with mildly incompetent lips and average nasolabial angle.

Radiographic Examination (OPG) revealed missing third molars in all quadrants excepting lower left third molar region which showed developing tooth bud. The periodontal status was healthy with no loss of lamina dura.

Examination of lateral cephalogram demonstrated an Orthognathic maxilla and mandible with class I skeletal base (ANB-2 degrees) and an average growth pattern (Go-Gn-Sn 31 degrees, FMA 26 degrees). The upper incisors were proclined and spaced (U1-NA 40 degrees/9mm, L1-NB 37 degrees/9mm).
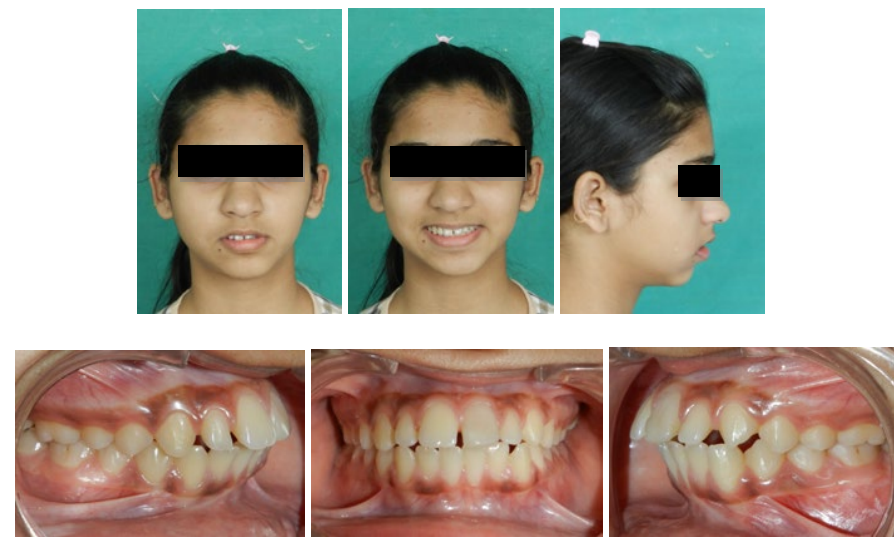

Figure 1. Pretreatment photographs.

Correspondence to: Ashwith B Hegde, Department of Orthodontics, M.A.Rangoonwala Dental College, Azam Campus, Camp Pune 411001, India, Tel: 09422027777; E-mail: orthoak47@gmail.com

Key words: esthetics, peg shaped lateral, laminate, spacing

Received: February 28, 2015; Accepted: April 04, 2015; Published: April 08, 2015 


\section{Diagnosis}

The patient had Angles Class I molar and canine relationship on right and left side with proclined and spaced upper incisors. The patient had average growth pattern with peg shaped lateral incisors on right and left side and there was mild crowding in lower anterior region.

\section{Treatment objectives}

- To achieve proper alignment.

- To achieve ideal overbite and overjet.

- To restore the peg shaped lateral incisor tooth to the normal shape and size.

- To close the spaces present in the upper arch and to correct lower incisors crowding.

- To Reduce lip strain and improve competency.

- 6 To achieve perfect soft tissue drape.

\section{Treatment plan}

Treatment was started with leveling and alignment of upper and lower teeth followed by retraction of upper and lowers anterior teeth and to restore the upper right and left peg shaped lateral teeth with the full ceramic crowns.

\section{Treatment Sequence:-}

Upper and lower arches were banded and bonded with .022 preadjusted edgewise appliance with MBT prescription (Figure 2). Initial leveling and alignment was achieved with the upper and lower $0.016 \mathrm{NiTi}$, then the closure of spaces was achieved with the $0.018 \mathrm{AJ}$ Wilcock wire and the space was maintained for the peg shaped lateral incisor to be replaced. The orthodontic treatment was completed in duration of one and half years (Figures 2 and 3).

In the next stage the patient was sent for the Prosthodontic rehabilitation and after crown preparation the upper right and left lateral incisors was restored with the full ceramic crowns (Figure 4).
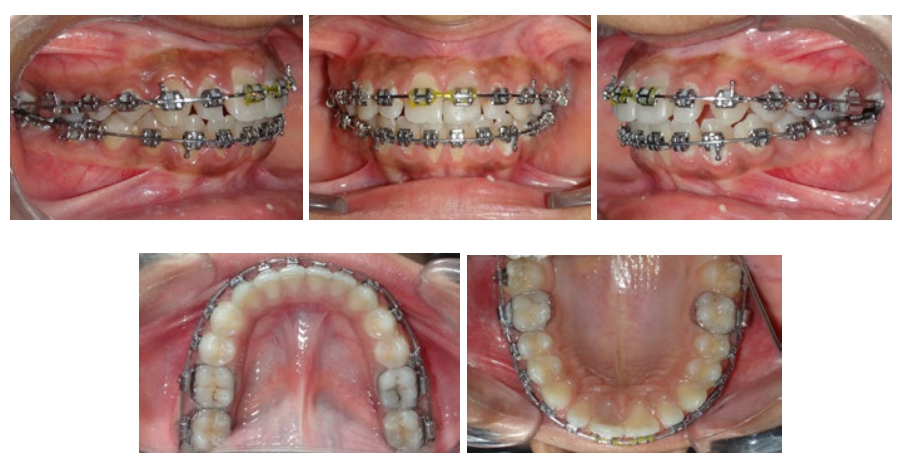

Figure 2. Intra-oral photographs during orthodontic treatment
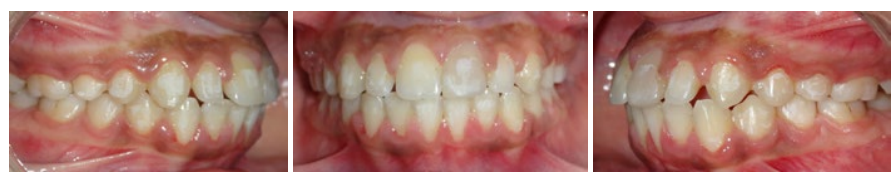

Figure 3. Intra-oral photograph of patient after orthodontic treatment.
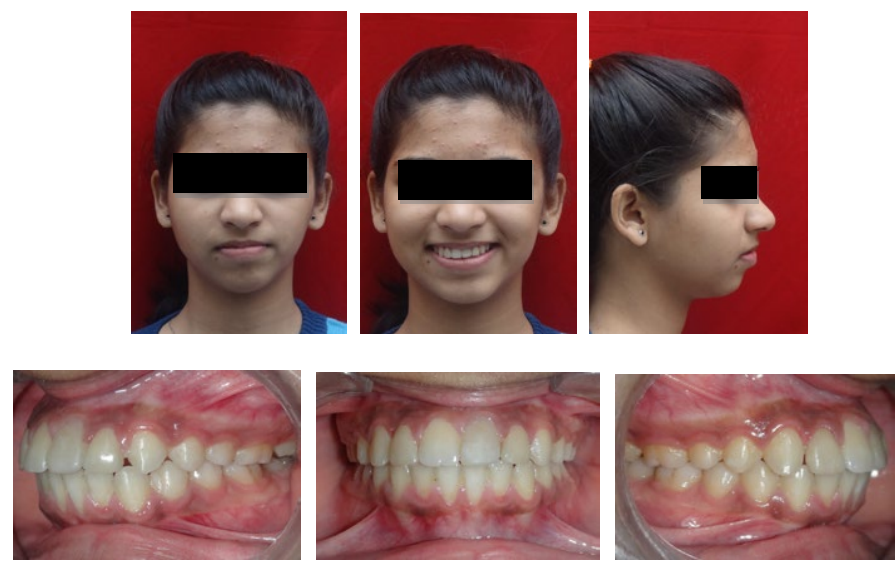

Figure 4. Photograph of the patients at the end of the prosthodontic treatment.

\section{Discussion}

Certain malocclusions particularly those seen in adults with deformed and mutilated dentitions defy cephalometrics based diagnostic algorithms. A small portion of orthodontic patient population will have some combination of missing and/or malformed lateral incisors [4]. Treatment of the condition is important to improve appearance, mastication and speech. Depending on the age it may cause severe psychologic disturbances [5]. We have to look very closely at these patients to reach a conclusion as each and every one of them is truly unique. Successful aesthetic orthodontic treatment demands not only an extensive knowledge of the evidence-based literature, but a tremendous level of creativity which is possible by involving other dental specialties. The current case describes an admirable level of artistry which is required in correction of the malocclusion and restoration of a functional occlusion with an aesthetic outcome. A team approach enhances the evidence-based knowledge and artistic creativity we possess.

\section{Conclusion}

A well-coordinated effort of interdisciplinary team resulted in a remarkable improvement in facial aesthetics smile and occlusal function.

\section{References}

1. Chan MD (1997) An adult malocclusion requiring a combination of orthodontic and prosthodontic treatment. Am J Orthod Dentofacial Orthop 111: 100-105. [Crossref]

2. Miller WB, McLendon WJ, Hines FB 3rd (1987) Two treatment approaches for missing or peg-shaped maxillary lateral incisors: a case study on identical twins. Am J Orthod Dentofacial Orthop 92: 249-256. [Crossref]

3. Nestel E, Walsh JS (1988) Substitution of a transposed premolar for a congenitally absent lateral incisor. Am J Orthod Dentofacial Orthop 93: 395-399. [Crossref]

4. Segura JJ, Jiménez-Rubio A (1998) Concomitant hypohyperdontia: simultaneous occurrence of a mesiodens and agenesis of a maxillary lateral incisor. Oral Surg Oral Med Oral Pathol Oral Radiol Endod 86: 473-475. [Crossref]

5. Winstanley RB (1984) Prosthodontic treatment of patients with hypodontia. J Prosthet Dent 52: 687-691. [Crossref]

Copyright: (C2015 Hegde AB. This is an open-access article distributed under the terms of the Creative Commons Attribution License, which permits unrestricted use, distribution, and reproduction in any medium, provided the original author and source are credited. 